\title{
A review of some measurements of muscle wasting, tone and clonus in paraplegia
}

\author{
E G Walsh MD FRCP FRSE
}

Department of Physiology, University of Edinburgh, Edinburgh, Scotland.

\section{Introduction}

The concept of muscle tone has been used in connection with physiological and pathological processes over many years but it is only recently that adequate methods have become available for quantitative determinations. Some of the work I am reviewing was undertaken jointly with $\mathrm{Mr}$ Phillip Harris, and with A J Douglas, P Edmond, G W Wright and G H Creasey. Clinical observations were made at the Spinal Unit, Edenhall Hospital, the instrumentation at the Department of Physiology, Edinburgh University and the Bio-Engineering Unit at the Princess Margaret Rose Hospital.

For determinations of tone it has been found to be much more satisfactory to make measurements of the motion induced by varying the force applied to the limb rather than by varying the position of the limb and recording the resultant force. The reasons for this have been discussed at length by myself in a monograph which has recently been published. ${ }^{1}$ A brief résumé of some of the arguments has been published in this journal. ${ }^{2}$

The most convenient way of applying controlled forces to a limb is by means of a 'printed motor'. Such a device, the moving parts of which are quite light, can convert electrical currents into the corresponding mechanical force instantaneously. Thus by controlling the current by a waveform generator a sequence of forces can be generated. These may be rhythmic or low frequency and abruptly alternating. The motion can be recorded by a potentiometer coupled to the motor and a signal corresponding to velocity obtained. At the same time additional data can be obtained by electromyography.

Different versions of the apparatus have

Correspondence: 64, Liberton Drive, Edinburgh EH16 $6 \mathrm{NW}$, Scotland. been used for measuring tone at a number of different sites. Many observations have been made at the wrist in normal subjects and in patients with neurological abnormalities and some observations have been made in hypermobile women. The apparatus is illustrated in Figure 1. With modifications suitable for the scale of the limb more limited observations have been made at the elbow and hip. In all of these investigations the motion has been in the horizontal plane. This excludes the effects of gravity which would change as the motion ensued and the angle changed.

In these various studies it has been found that the limb exhibits a resonance, that is to say that at a certain frequency of excitation the motion is greatest. This is the resonant frequency and it is a value quite important for estimating muscle tone. The relationship which is relevant is:

$$
f=\frac{1}{2 \pi} \sqrt{\frac{K}{J}}
$$

where $f$ is the resonant frequency, $K$ the stiffness and $J$ the inertia. It is thus apparent that the square of the resonant frequency reflects stiffness.

\section{Muscle wasting}

Observations were made at the knee in cases of complete transection of the spinal cord. All of the patients had reached a plateau in their status, the lesions being between 6 months and 10 years old. The measurements were made both before and after a course of electrical stimulation of the thigh muscles undertaken for therapeutic reasons. Monophasic rectangular pulses of $0.3 \mathrm{~ms}$ duration at $20 \mathrm{~Hz}$ were used. The duty cycle was $4.5 \mathrm{~s}$ on and $4.5 \mathrm{~s}$ off. The stimulation was for up to $1 \mathrm{hr}$ per day for 5 days per week. Training lasted for at least 2 months. The age of the patients was $31.3 \pm$ 


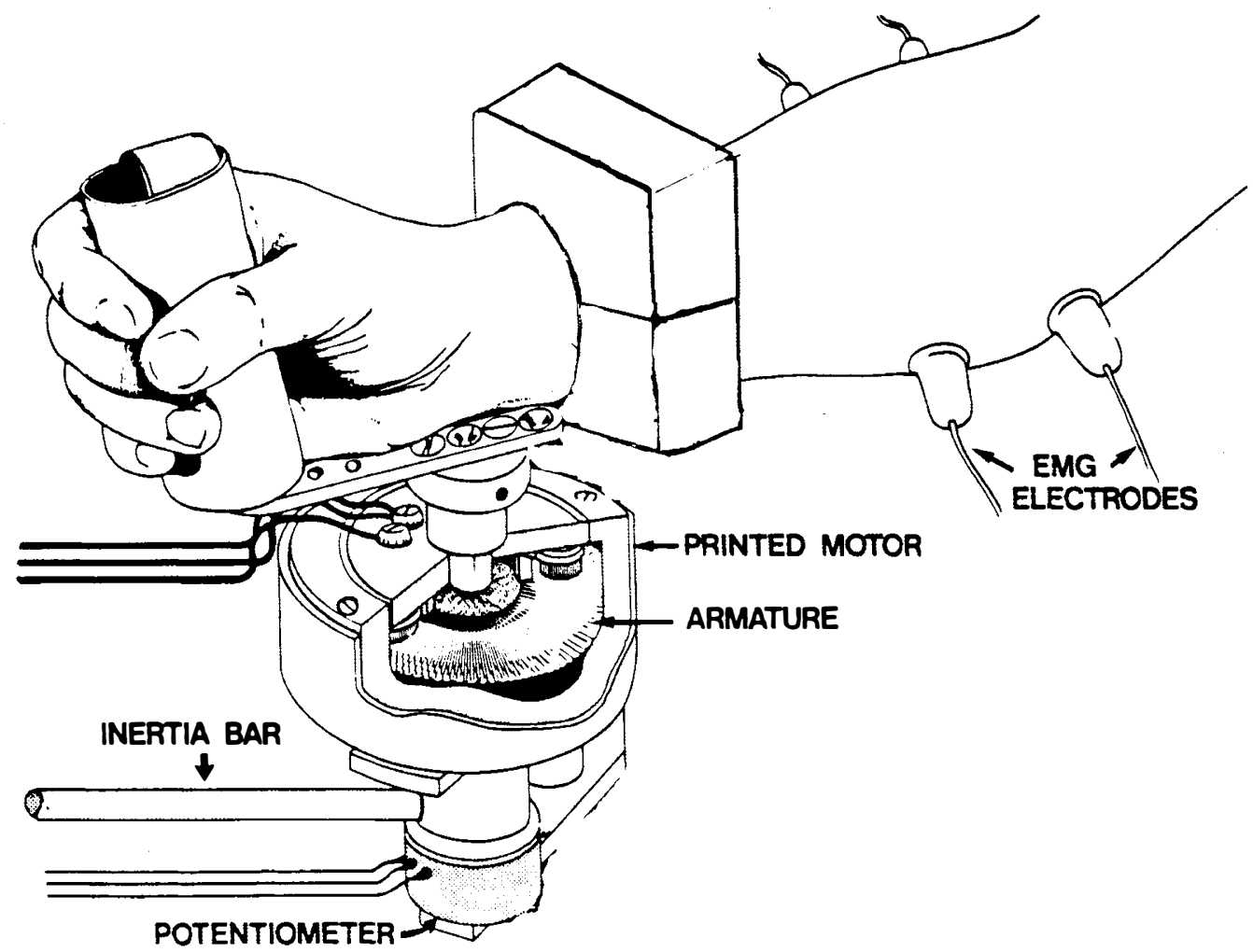

Figure 1 Arrangements for testing muscle tone of the forearm by applying rhythmic forces around the wrist. The cut away representation of the housing of the motor allows for a view of the armature. It is useful to record the surface emg, electrodes are shown. For special investigations the inertia may be increased by attaching a metal bar to the apparatus. ${ }^{3}$

2.0 years. Controls were healthy hospital personnel age $27.7 \pm 2.2$ years.

There is some wasting of the musculature in paraplegia and measurements made in this series confirmed this well known fact. Data about thigh circumferences are given in Figure 2. There was some increase of bulk after electrical stimulation.

\section{Muscle tone at the knee}

The measurements of muscle tone were undertaken on the same group of paraplegics and controls as those whose data about thigh circumference are given above. The apparatus is shown in Figure 3. Here, as at other joints in normal subjects, a resonance was found. The motion was greatest when the driving force was at about $0.5 \mathrm{~Hz}$ if the motion was of moderate degree.

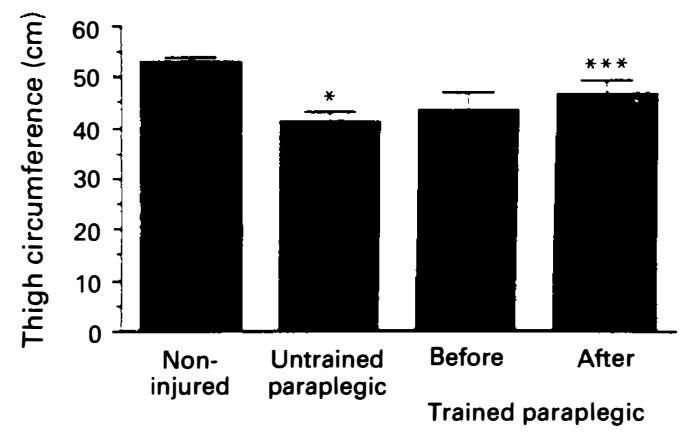

Figure 2 Thigh circumference in control subjects $(n=14)$, established paraplegia $(n=1)$, and paraplegics who have been subjected to a course of electrical stimulation $(n=5)$. Values are means \pm SEM. ${ }^{*} p<0.001$ compared to controls (Student's $t$ test). ${ }^{* * *} p<0.001$ compared to results before the stimulation programme. 4 


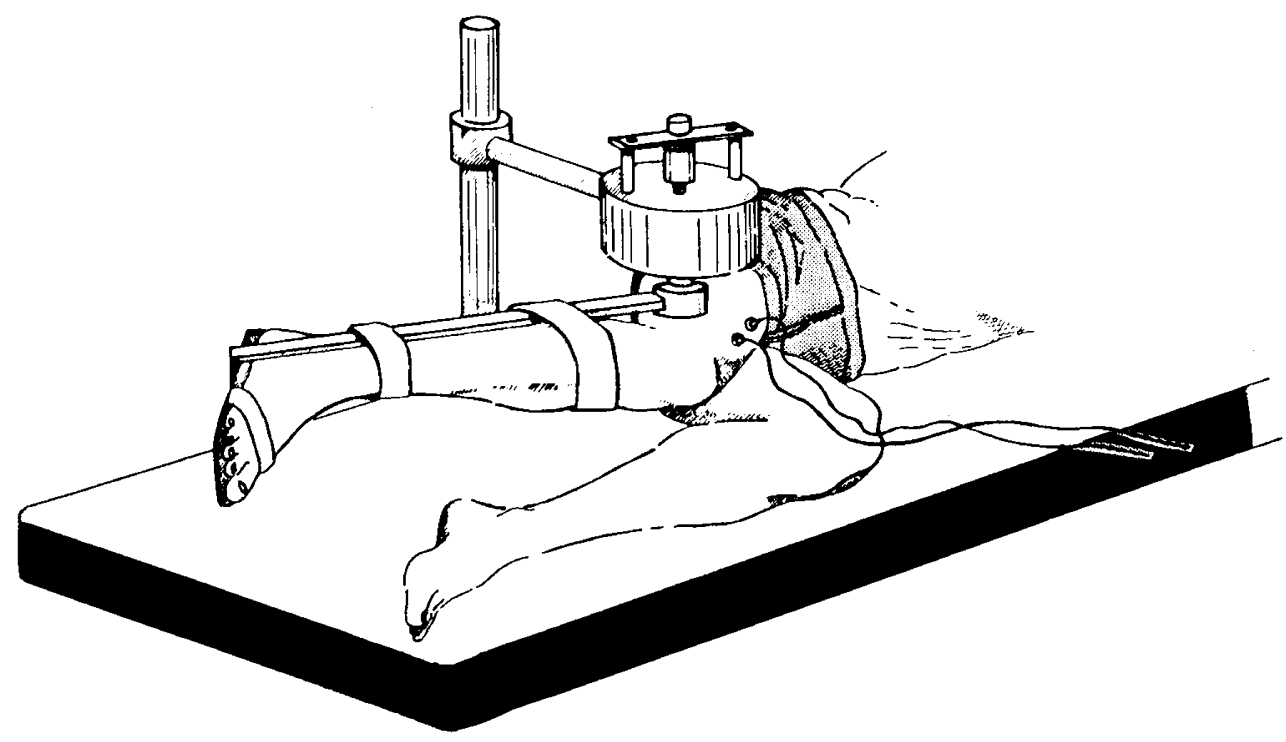

Figure 3 Arrangements for testing muscle tone at the knee. The printed motor is concentric with the joint. Surface electrodes over the quadriceps are shown. ${ }^{5}$

In these patients too a resonance was found (Fig 4). It was naturally assumed that muscle tone would be higher in paraplegics. The results however did not show that; rather the values are the same or lower. When there had been treatment by electrical stimulation the curves were virtually the same as those of the control subjects (Fig 5).

The motion of a limb in response to a force is constrained not only by the stiffness of the muscles but also by damping which represents forces of a viscous nature related to the velocity of the movement. The damping is another factor to be considered when using the all-inclusive, global term 'muscle tone'. Damping may be estimated from measurements of the peak velocity at resonance for, under that circumstance, the effects of limb inertia and muscle stiffness are equal and opposite and cancel out. The results are shown in Figure 6. At most of the values the limbs of the paraplegic subjects have less damping than those of the controls, ie they move more for a given rhythmic force. Again there is no evidence of increased muscle tone.

Concordant results were obtained by electromyography. The muscles were elec- trically silent except when the swings imparted by the motor were very wide.

\section{Spasms}

In some of the observations on resonant frequency, spasms were taken during a recording. These occurred predominantly when very wide swings of the leg occurred at resonance with the higher values of driving torque.

Following a period of electrical stimulation too the muscles may remain temporarily contracted as is shown by a rise in resonant frequency (Fig 4).

Other observations on spasms were made in a male paraplegic telephone operator aged 28. He had evolved for himself a manoeuvre by which he could get himself erect from his wheel chair. He thumped both thighs with his fists and there resulted an extensor spasm of the knees (Fig 7). There appeared to be a clonic state which merged into long lasting steady contraction.

\section{Ankle clonus}

Clonus is a common sign in paraplegia and other neurological conditions and it is at 
Sinusoidal chirps (at increasing frequency)
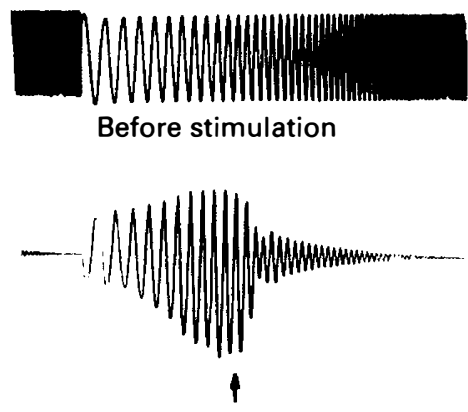

Sinusoidal chirps
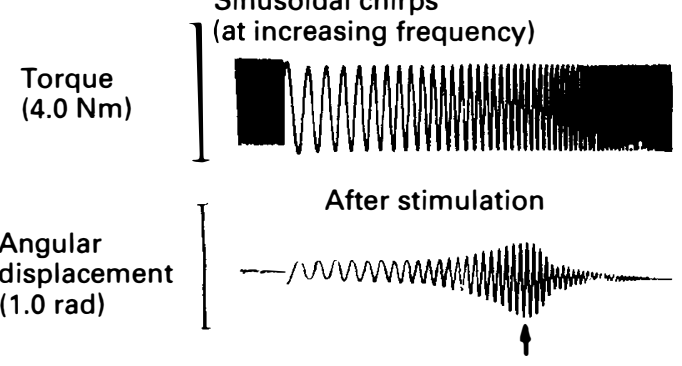

Figure 4 The torque applied to the knee has a frequency which increases exponentially. At a certain rate the motion is greatest. This is the resonant frequency and reflects muscle stiffness. After electrical stimulation, in the instance shown, the resonant frequency is greatly increased due to the occurrence of a spasm. ${ }^{4}$

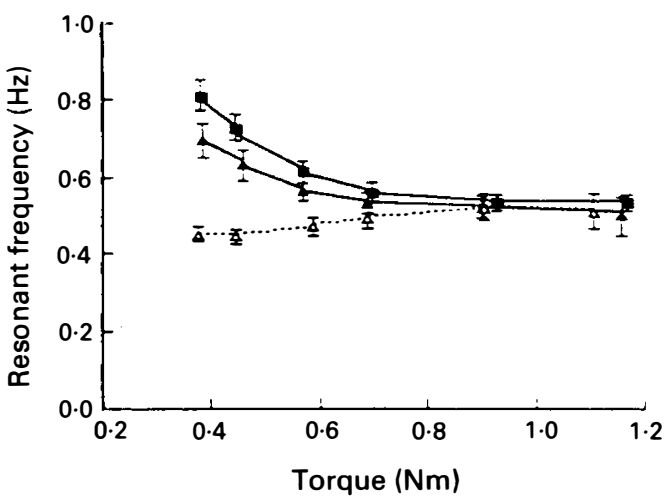

Figure 5 Graphs of resonant frequency at different values for the peak to peak rhythmic torque. Filled squares - normal subjects, hollow triangles - untreated paraplegics, filled triangles - paraplegics who have been treated by electrical stimulation. 4

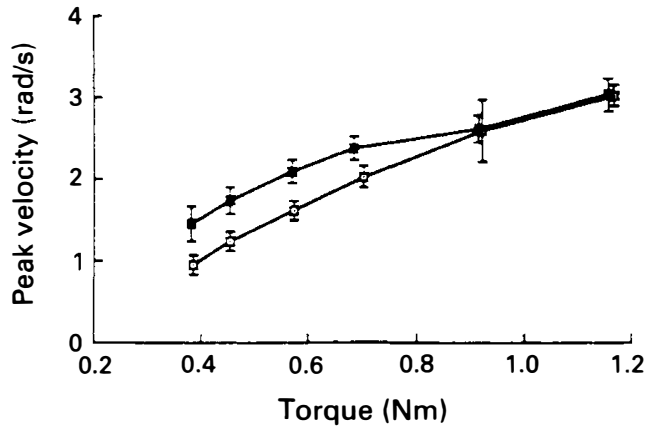

Figure 6 Peak velocity at resonance at different torque levels. Hollow squares - normal subjects. Filled squares - paraplegics. ${ }^{5}$
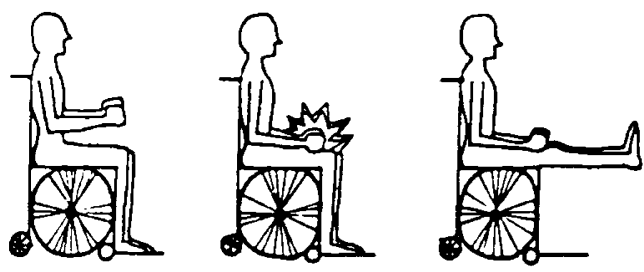

Figure 7 Procedure by which a paraplegic found he could rise to a standing position. ${ }^{6}$

times quite disturbing to the patient, as for instance when the vibrations interfere with reading. It has however been the subject of very little study.

For these measurements the apparatus used is shown in Figure 8. Again a printed motor, which is concentric with the ankle joint, is used.

Nine patients were investigated in 6 of whom there had been damage to the spinal cord. In all a dorsiflexing force was needed to bring out the clonus and the rate was never slower than 5 or faster than $8 \mathrm{~Hz}$. The current through the motor was thus used to duplicate the manoeuvre of a clinical examination where the physician presses upwards on the ball of the foot. The torque needed was much less than that exerted on the ankle during normal walking. If the bias was applied gradually the clonus did not start at once but took some seconds to build up. If the conditions were then held constant clonus continued with each beat closely following the pattern of its predecessors. In this almost machine-like regularity clonus 


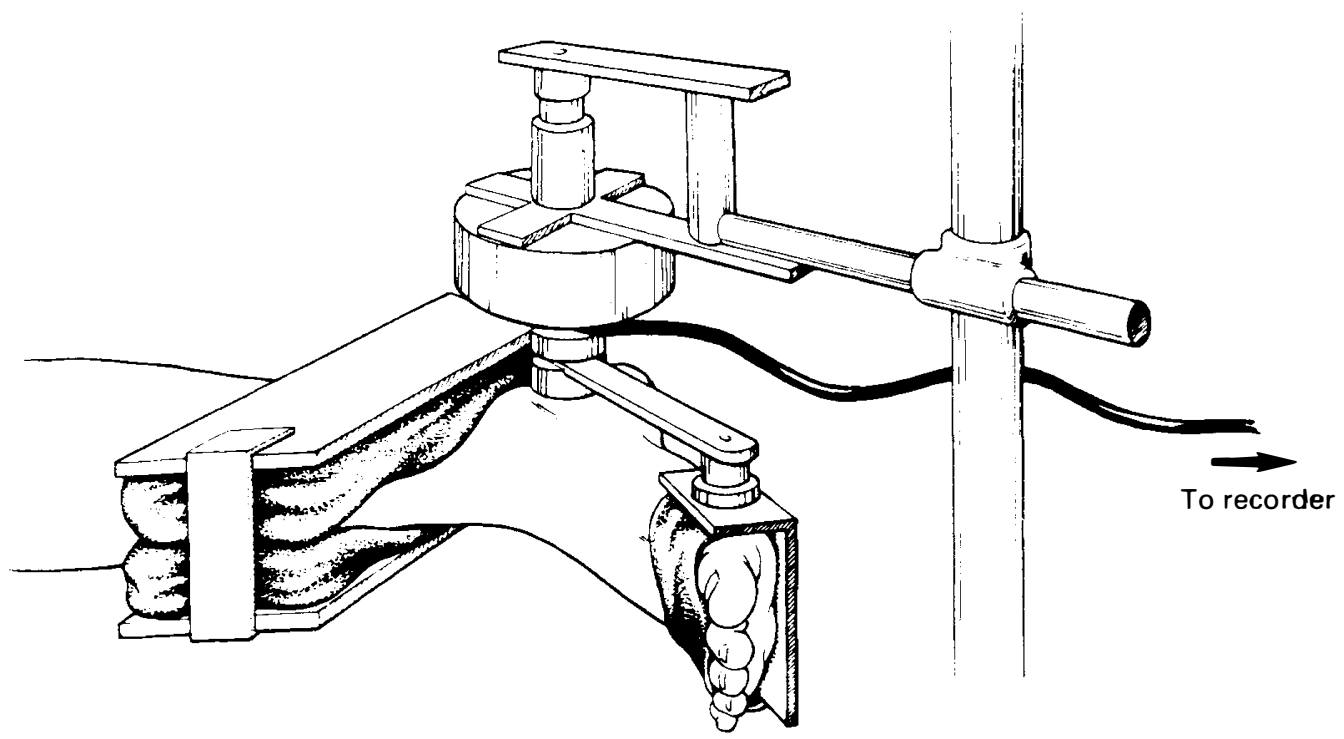

Figure 8 Arrangements for making biomechanical measurements at the ankle.

differs from tremor which even at its most uniform expression, varies significantly in amplitude. Clonus is more jerky than a rhythmic voluntary act and it is difficult to see it in terms of Hughlings Jackson's concept of the release from inhibition of a fragment of normal motor performance.

The contraction of clonus is abrupt, the onset and cessation of electromyographic activity being more clear than, for example, in the tremor of Parkinsonism. The amplitude varies considerably according to the level of dorsiflexing force applied but the frequency is essentially constant. When the force is withdrawn the motion ceases at once but the rhythmic electromyographic discharges may continue for a number of beats (Fig 9). If the skin over the calf musculature is observed at that time small fluctuations in the contours may often be observed corresponding to the electrical discharges. As the mechanical conditions in the limb change very widely while the rate of discharges is invariable, it was suggested that

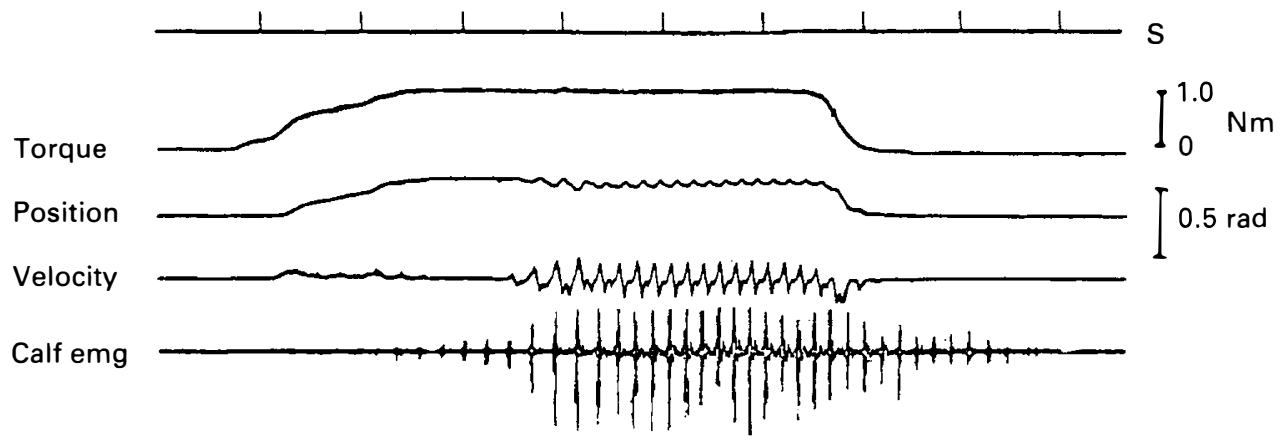

Figure 9 A dorsiflexing bias was controlled manually and gradually increased. Clonus started, when the biasing force was withdrawn the mechanical oscillations were no longer seen but electrical rhythmicity continued for a number of beats. Male patient aged 23, traumatic complete mid thoracic lesion. ${ }^{7}$ 
the clonus resulted from the discharge of a central pacemaker. The more popular hypothesis that clonus results from self re-excitation of hyperactive stretch reflexes does not afford a ready explanation of the phenomena observed.

In 5 patients rhythmic forces were generated by the motor in addition to the gentle dorsiflexing pressure. When the rate was close to but not identical with that of the clonus it was possible to set up beats, the size of the oscillations varying slowly at a rate dependent on the difference in the frequencies of the clonus and the rhythmic force (Fig 10). This was not a difficult procedure and could usually be accomplished by trial and error in a short time. The electromyographic discharges showed changes corresponding to the beats, the activity being greatest when the swings were large.

\section{Thixotropic stiffening}

The term thixotropy is not at present widely known. It is discussed at length in my recent monograph $^{1}$ and, more briefly, in this journal. ${ }^{8}$ In the connection of posture it refers to the changes of stiffness of a muscle that are shown when it is at rest. Following a period of motion which may be quite brief (eg a single movement) the muscle is comparatively flexible. With inactivity it stiffens at first rapidly, then more slowly. This stiffening is more difficult to loosen if the muscle is cold.

The limbs of paraplegic patients are commonly immobile for long periods compared with the almost constant intermittent minor movements of a healthy person. They are also likely to be comparatively cold. Both of these factors will increase, it may be assumed, the extent of thixotropic stiffening.

I had the opportunity of making some measurements on a tetraplegic patient with a complete lesion at C7. Using the apparatus illustrated above (Fig 3 ) very clear thixotropic effects were observed at the knee. An example of the results is shown in Figure 11. Clearly further study of this phenomenon in paraplegic patients is called for. Measurements of the effect are likely to be of value in comparing the efficacy of different regimes of physiotherapy.

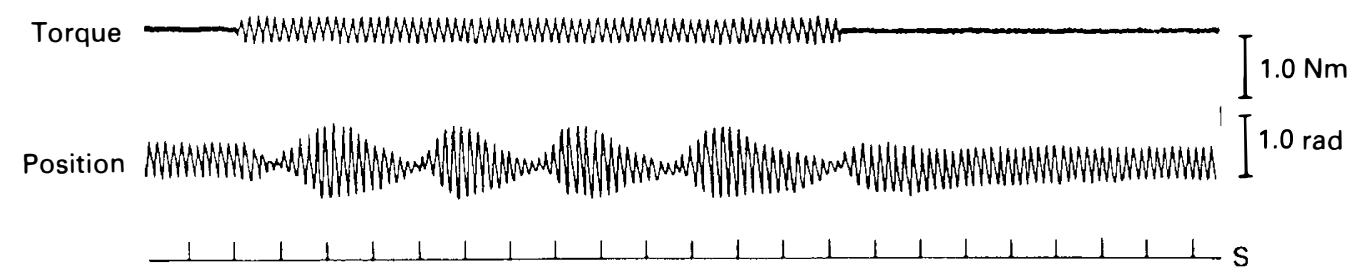

Figure 10 Beats set up between a rhythmic force and established clonus. Same subject as Figure 9.7

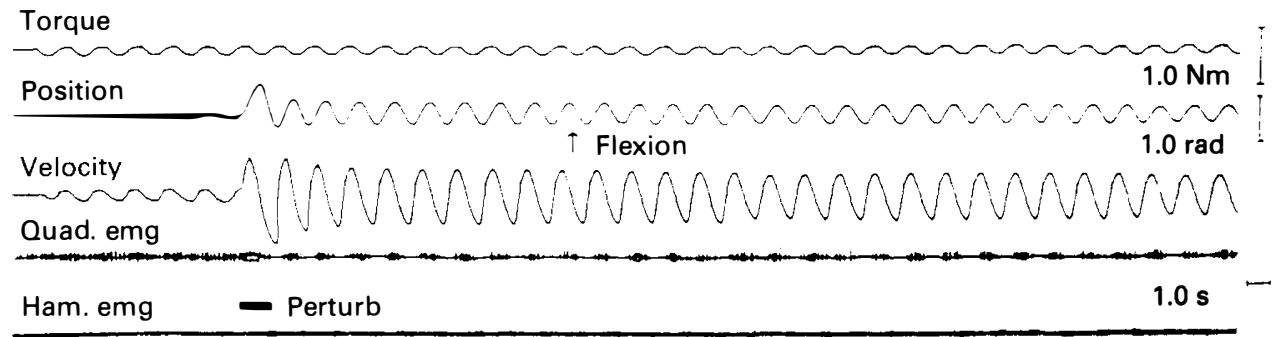

Right knee

Figure 11 The motor generates an oscillatory torque at about $1 \mathrm{~Hz}$. The oscillations of the paralysed limb are greatly increased after the perturbation produced by moving the limb by hand. ${ }^{8}$ 


\section{References}

1 Walsh EG (1992) Muscles, Masses and Motion. The physiology of normality, hypotonicity, spasticity and rigidity. MacKeith, London; Blackwells, Oxford; Cambridge University Press, New York.

2 Walsh EG (1992) The measurement of muscle tone. Paraplegia 30: 507-508.

3 Lakie M, Walsh EG, Wright GW (1984) Resonance at the wrist demonstrated by the use of a torque motor: an instrumental analysis of muscle tone in man. J Physiol 353: 265-285.

4 Douglas A, Walsh EG, Wright GW, Creasey GH, Edmond P (1991) The effects of neuromuscular stimulation on muscle tone at the knee in paraplegia. Exper Physiol 76: 357-367.

5 Douglas A, Walsh EG, Wright GW, Edmond P (1989) Muscle tone around the human knee in paraplegia. $Q$ J Exper Physiol 74: 897-905.

6 Harris P, Walsh EG (1972) Simultaneous electrical and mechanical recording from postural muscles in a paraplegic patient. Paraplegia 9: 229-230.

7 Walsh EG (1976) Clonus: beats provoked by the application of a rhythmic force. J Neurol Neurosurg Psychiatr 39: 266-274.

8 Walsh EG (1992) Postural thixotropy: a significant factor in the stiffness of paralysed limbs? Paraplegia 30: $113-115$. 